\title{
Performance of tRPC prototypes with relativistic heavy ions
}

\section{Carlos Paradela', Yassid Ayyad, José Benlliure, Ignacio Durán}

University of Santiago de Compostela

Facultad de Física, E-15782, Spain

E-mail: carlos.paradela@usc.es

\section{Enrique Casarejos}

University of Vigo, E-36310, Spain

In this work we report on the time performance of single-gap and double-gap timing Resistive Plate Chambers (tRPCs) with $10 \mathrm{MeV}$ electron bunches at the electron accelerator of the CEA/DAM/DIF (Commissariat à l'énergie atomique, Military Applications Division, Île de France) and with $500 \mathrm{~A} \cdot \mathrm{MeV}{ }^{136} \mathrm{Xe}$ ions accelerated by the SIS18 synchrotron at GSI (Helmholtzzentrum für Schwerionenforschung). The measurements with electrons show a clear dependence of the time resolution with the beam intensity, reaching values below $30 \mathrm{ps}(\sigma)$. On the other hand, a time resolution of about $45 \mathrm{ps}$ is obtained for the single-gap tRPCs with the xenon beam, but it worsens when we increase the event rate above several $\mathrm{Hz} / \mathrm{cm}^{2}$.

XI workshop on Resistive Plate Chambers and Related Detectors (RPC2012)

INFN-Laboratori Nazionali di Frascait, Italy

February 5-10, 2012

\footnotetext{
$1 \quad$ Speaker
} 


\section{Introduction}

tRPCs have been proposed for building a time-of-flight (ToF) wall for detecting relativistic heavy ions at the $\mathrm{R}^{3} \mathrm{~B}$ (Reactions with Relativistic Radioactive Beams) experiment at FAIR (Facility for Antiproton and Ion Research) [1]. In order to identify nuclei around mass number 150, a time resolution of $50 \mathrm{ps}$ is required for the ToF wall tRPCs. The full requirements of this detector are described with more detail in [2]. The time resolution of tRPCs has been extensively investigated with minimum ionizing particles (MIPs), achieving values below 50 ps even for large area chambers [3]. However, their performance with relativistic ions is scarcely known. Recently, we have shown that the efficiency for detecting ions heavier than alphas is close to $100 \%$ with single and double-gap RPCs [4]. In this work we will report on the time resolution obtained in measurements with electron bunches and relativistic heavy ions.

\section{RPC prototypes}

The measurements here reported were performed with two different prototypes. The first one consists in small-size symmetric double-gap tRPCs in which the electrodes are made of four commercial float glass of $1 \mathrm{~mm}$ thickness with self-adhesive copper tape disposed as a single strip of $2 \mathrm{~cm}$ width in between the two inner glasses and in the external sides of the stack [4]. The gas gaps are defined by using $300 \mu \mathrm{m}$ thickness fishing line. High voltages of 85-110 $\mathrm{kV} / \mathrm{cm}$ are typically applied, while the isobutane-free gas mixture is composed of $\mathrm{C}_{2} \mathrm{H}_{2} \mathrm{~F}_{4}(90 \%)$ and $\mathrm{SF}_{6}(10 \%)$. The signal readout is done in two stages: the one that is next to both strip ends consists in an own-developed amplifier based on two commercial chips: the BFR92A 5GHz wideband transistor and the MAX4224 current amplifier (600 MHz nominal bandwidth), with a RC circuit adapting the strip impedance. The second stage is based on the Front-End Electronics

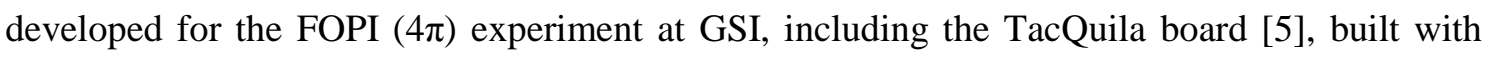
high-resolution Time-to-Amplitude Converter ASIC chips, and a QDC board mounted on the TacQuila card, that provides the signal charge. The time resolution of the FOPI acquisition amounts up to $25 \mathrm{ps}$. In order to carry out the timing studies, we have built a detection system including two identical RPCs. They are placed inside a gas-tight aluminium box, mounted along the box bottom with a separation a few centimeters as it is shown in the left picture of Fig. 1. Thin plastic windows are manufactured in the box cap and bottom in order to reduce the energy straggling of the ions. During the tests, the detection setup is placed orthogonally to the beam and the time differences between both tRPCs can be used to obtain the time resolution as we will explain in next section. 

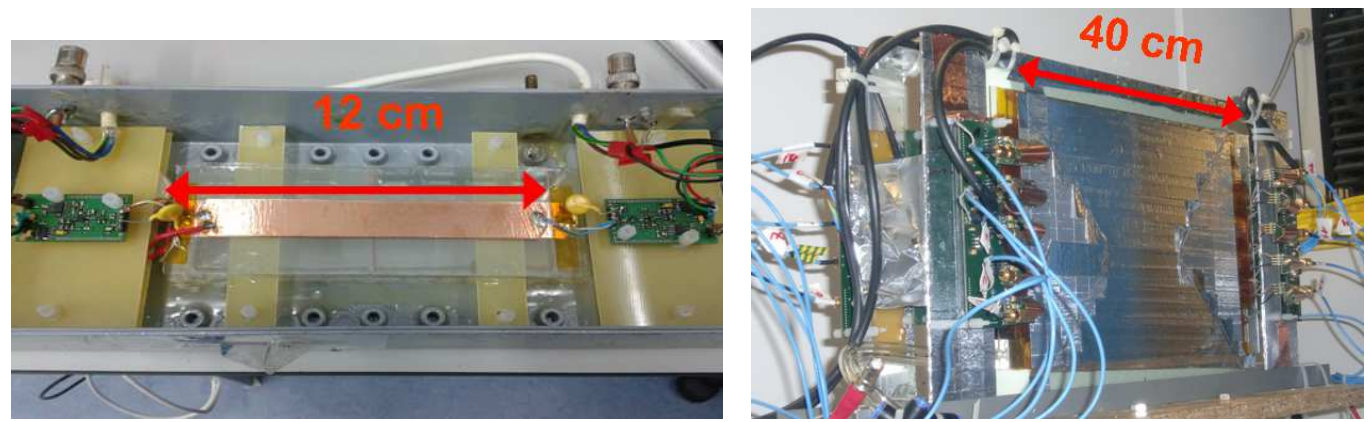

Fig. 1: Double-gap (left) and single-gap (right) tRPC prototypes described in this work.

The second tRPC prototype that we have tested is based on the single-gap concept. The structural materials and dimensions are kept from the double-gap prototype except that a larger detection surface has been build to implement five strips of $40 \mathrm{~cm}$ length. The separation between strips is $2 \mathrm{~mm}$. Another important difference is that the gas volume is defined by the glass electrodes themselves, being their perimeter completely glued to assure the tightness, except in two points where special adapters to the gas pipes has been designed to keep the gas flow. Similar electronics have been used for this prototype, replacing the MAXIM chip by the OPA657 amplifier with a $1.6 \mathrm{GHz}$ bandwidth. Two single-gap RPCs are stacked together in such a way that the high voltage electrodes are facing each other and separated by several centimeters. Therefore, timing between both RPCs can also be measured with this configuration.

\section{Beam tests and results}

The previously described tRPC prototypes have been tested under different beam conditions in two international facilities: the ELSA electron accelerator of CEA/DAM at Bruyères-le-Chatel (France) and the heavy ion facility of GSI at Darmstadt (Germany).

\subsection{Test with electron beams}

Timing properties of our tRPC were studied, for the first time, by using an electron beam in the ELSA facility. Only the double-gap prototype was available at that time. $10 \mathrm{MeV}$ monoenergetic electrons were delivered in bunches of a few ps length. The idea was to simulate the energy lost by the heavy ions in the RPC by means of many electron losses in the same spot. Within this scope, the beam was collimated by using a lead block with a $2 \mathrm{~mm}$ diameter hole, just in front of the detectors. Therefore, a unique RPC signal was obtained for each electron bunch, being its charge correlated with the number of electrons in the bunch. This can be modulated by using a polarizer that varies the beam intensity in two orders of magnitude. Unluckily, the beam intensity could not be measured during the test, but from previous measuremenents we can estimate a full intensity of about $10^{4}$ electrons per bunch after the collimator. An accelerator operation mode of $5 \mathrm{~Hz}$ was used to keep low-rate conditions. In addition to the twin RPC chamber, a thin plastic scintillator connected to two fast 
photomultipliers provided by the CEA-SOFIA group [6] was placed in front of the RPCs that give us a high-resolution timing measurement in coincidence with the RPCs.

We have measured the time differences between both RPCs and also between each RPC and the thin plastic scintillator. The average time measured at both strip sides is used for calculating the time difference between detectors, which corresponds to the $10 \mathrm{MeV}$ electrons ToF. For determining the detector time resolution, the ToF dispersion can be neglected and the time difference spread is coming from the contribution of the detector time resolution. As we have measured three different ToFs, we can solve the equation system to obtain the intrinsic time resolution of each detector. These measurements have been done for different RPC voltages and beam intensities. The time resolutions obtained for the best performing RPC are shown in Fig. 2 compared to the resolution obtained with the thin plastic. The measured charge distributions are very narrow and no slewing correction has been applied in the data analysis. Moreover, time and charge distributions are quite clean and tail free. Time resolution depends strongly on the beam intensity, reaching values of $50 \mathrm{ps}$ for beam intensities of $2 \%$ and below $30 \mathrm{ps}$ for a intensity of $20 \%$, close to the acquisition system intrinsic resolution. On the other hand, resolutions hardly change for different RPC high voltages, leading to a broad operating plateau.

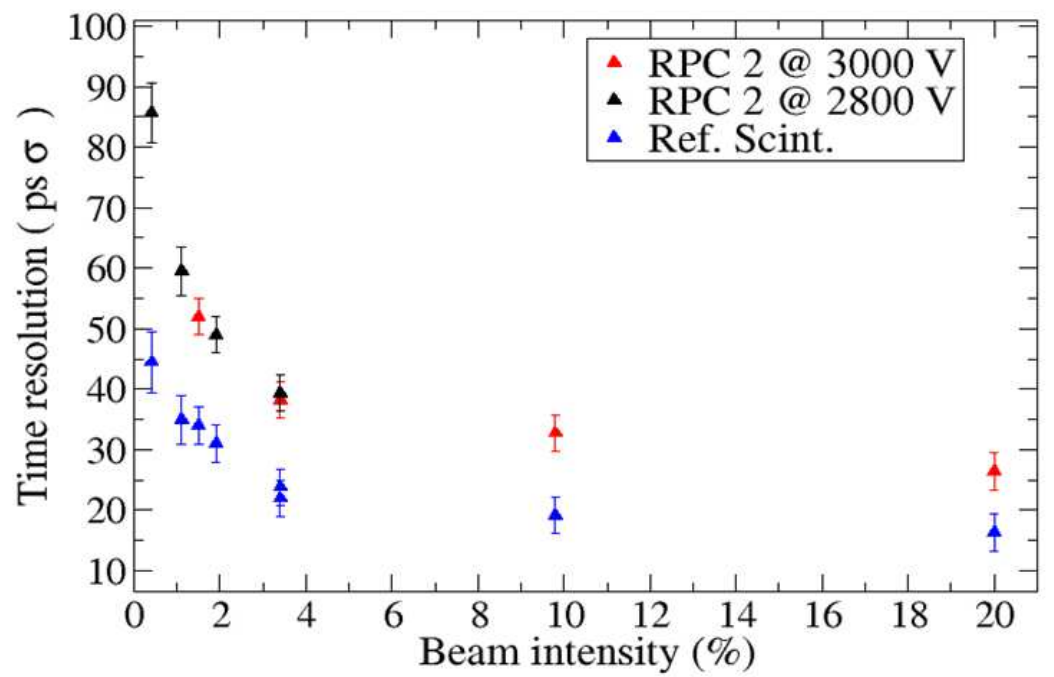

Fig. 2: Time resolutions measured for the second RPC in the stack and for the reference plastic scintillator are represented for different electron beam intensities. Two different RPC applied voltages are included to cover a larger intensity range.

\subsection{Test with the uranium beam}

The same double-gap tRPC setup was tested at the GSI facility by using a $1 \mathrm{~A} \cdot \mathrm{GeV}{ }^{238} \mathrm{U}$ beam. The trigger was provided by the RPCs themselves because no reference plastic could be included in the setup due to beam time and space limitations. Furthermore, because of the test conditions, it was difficult to decrease the beam intensity below $100 \mathrm{~Hz}$ and we only could measure a few runs with reduced beam rate (several tens of ions per second) and a beam spot of $2 \mathrm{~cm}$ diameter. Finally, an important contribution of scattered particles coming with the beam 
was observed, being suppressed by applying cuts in the RPC position distributions obtained from the time difference between both RPC sides. In the most favourable case, with the RPCs working at $2800 \mathrm{~V}$, the time distribution width obtained by fitting the ToF was 96 ps which correspond to a time resolution of 68 ps when considering that both RPC resolutions are equal. In this analysis, two slewing corrections were applied by using second order polynomials to take into account the time dependence with the charge in both detectors.
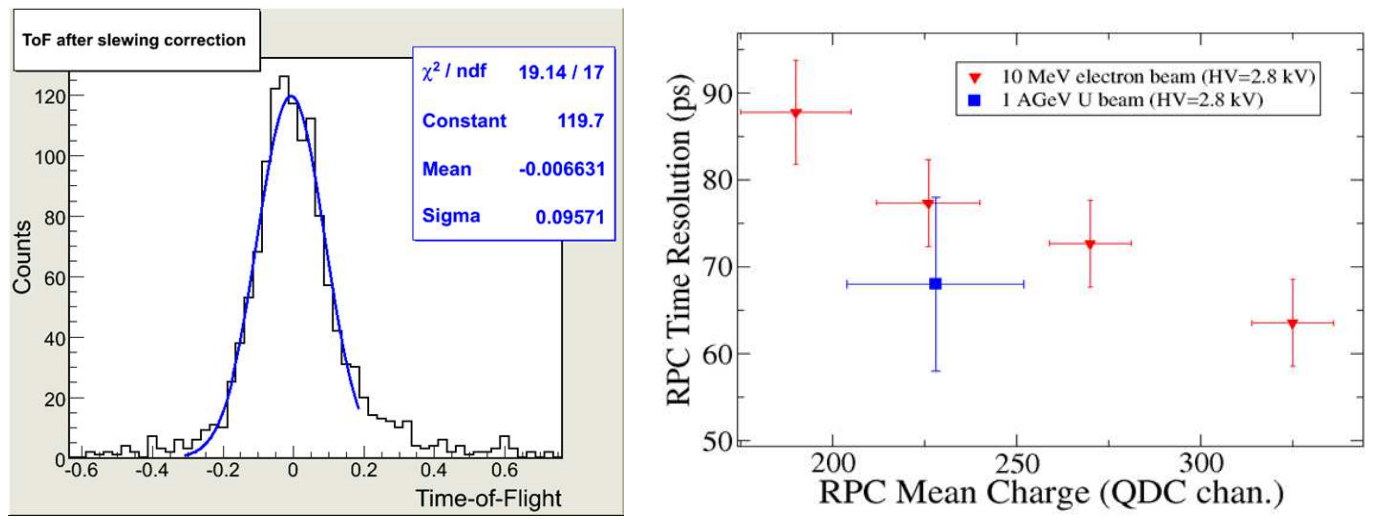

Fig. 3: ToF distribution obtained for the double-gap tRPCs with the uranium beam (left chart) and the time resolution represented in function of the mean charge value (right chart).

The results obtained for electrons with similar signal charge are plotted for comparison.

\subsection{Test with the xenon beam}

More recently, the single-gap tRPC prototype was tested at the GSI facility by using a 500 $\mathrm{A} \cdot \mathrm{MeV}{ }^{136} \mathrm{Xe}$ beam. This time, better experimental conditions were achieved: two reference plastic scintillator in coincidence provided a RPC external trigger and beam rates as low as 10 $\mathrm{Hz}$ were obtained from the synchrotron during long time. Therefore, we can study the RPC time resolution in an extended voltage range. As it was done in the previous test, the time resolution is obtained from the ToF between the two RPCs in the stack, considering that both RPCs have the same intrinsic resolution. Under this assumption, we have obtained, after a preliminary analysis, values below 50 ps for certain applied voltages as it is shown in the left chart of Fig. 4. Because of the synchrotron operation mode at such a low rate, this was largely fluctuating between different spills. Luckily, thanks to the calibration mode of the acquisition system, we can include the trigger rate in the analysis and the rate effects in the resolution were studied by splitting the data in two rate intervals. As we can observe in the second chart of Fig. 4, an important worsening of the resolution is observed with increasing rates, even for a reduced rate of several $\mathrm{Hz} / \mathrm{cm}^{2}$. In any case, the time resolution is spoiled for applied voltages beyond 3000 $\mathrm{V}$ what could be explained by the increasing contribution of streamers. 

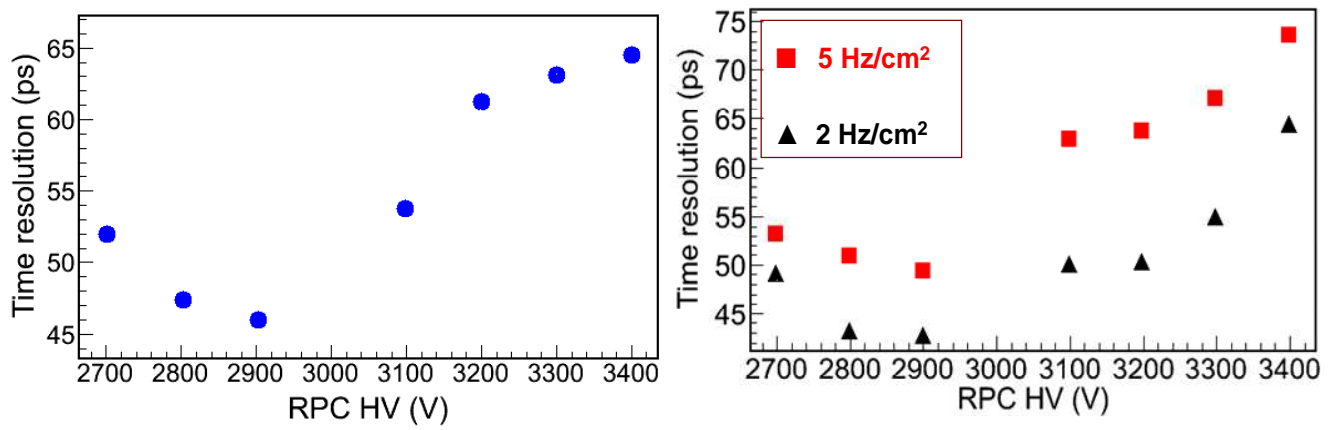

Fig. 4: Time resolution measured with the single-gap prototype in a ${ }^{136} \mathrm{Xe}$ beam for different RPC high voltages. Time resolution has been studied considering all the statistics available together (left chart) and then splitting it according to the counting rate (right chart).

\section{Conclusions}

In this work, the time resolution of double-gap and single-gap tRPC prototypes has been studied by using electron and heavy ion beams. For both prototypes, we have measured time resolutions below $50 \mathrm{ps}$. In the case of electron bunches, the time resolution improves below 30 ps for the highest beam intensities and the results are compatible with ions when we compare similar signal charges. The best results with ions, $\sigma_{T}=43 \mathrm{ps}$, have been obtained for the singlegap prototype with a very low rate ${ }^{136} \mathrm{Xe}$ beam $\left(2 \mathrm{~Hz} / \mathrm{cm}^{2}\right)$. However, it has been observed that the rate effects with heavy ions become relevant at much lower rates than for MIPs.

\section{References}

[1] H. Alvarez-Pol et al., Nucl. Phys. B Proc. Supp. 158 (2006) 186

[2] E. Casarejos et al., Structural design of the RPC-based ToF wall (iTOF) for the R3B experiment at FAIR, in these proceedings [PoS(RPC2012)074].

[3] A. N. Akindinov et al., Nucl. Instrum. Meth. A 602 (2009) 658

[4] E. Casarejos et al., Detection efficiency of relativistic heavy-ions with resistive-plate chambers, Nucl.Instrum. Meth.A 674 (2012) 39

[5] K. Koch et al., IEEE Trans. Nucl. Sci NS-54(4) (2005) 1201

[6] A. Bail et al., Time of flight measurement on the SOFIA experiment, ANIMMA 2011 Conference Proceedings. 